ZU-TH 29/07

PITHA-07/17

Edinburgh 2007/42

SLAC-PUB-13015

November 2007

\title{
Master Integrals for Fermionic Contributions to Massless Three-Loop Form Factors
}

\author{
G. Heinrich ${ }^{a}$, T. Huber ${ }^{b, c}$ and D. Maître ${ }^{d}$ \\ ${ }^{a}$ School of Physics, The University of Edinburgh, Edinburgh EH9 3JZ, Scotland, UK \\ ${ }^{b}$ Institut für Theoretische Physik, Universität Zürich, Winterthurerstrasse 190, \\ CH-8057 Zürich, Switzerland \\ ${ }^{c}$ Institut für Theoretische Physik E, RWTH Aachen \\ D-52056 Aachen, Germany \\ ${ }^{d}$ Stanford Linear Accelerator Center, Stanford University, Stanford, CA 94309, USA
}

\begin{abstract}
In this letter we continue the calculation of master integrals for massless three-loop form factors by giving analytical results for those diagrams which are relevant for the fermionic contributions proportional to $N_{F}^{2}, N_{F} \cdot N$, and $N_{F} / N$. Working in dimensional regularisation, we express one of the diagrams in a closed form which is exact to all orders in $\epsilon$, containing $\Gamma$-functions and hypergeometric functions of unit argument. In all other cases we derive multiple Mellin-Barnes representations from which the coefficients of the Laurent expansion in $\epsilon$ are extracted in an analytical form. To obtain the finite part of the three-loop quark and gluon form factors, all coefficients through transcendentality six in the Riemann $\zeta$-function have to be included.
\end{abstract}




\section{Introduction}

The quark form factor $\gamma^{*} \rightarrow q \bar{q}$ and gluon form factor $H \rightarrow g g$ (effective coupling) are the simplest processes containing infrared divergences at higher orders in massless quantum field theory, and therefore appear in a large variety of physical applications. They can for instance be used to predict the infrared pole structure of multi-leg amplitudes at a given order $[1,2]$ and to extract resummation coefficients [3], and they are needed for the purely virtual corrections to a number of collider reactions (Drell-Yan process, Higgs production and decay, DIS).

At the two-loop level, corrections to the massless quark [4] and gluon $[5,6]$ form factors were computed in dimensional regularisation with $D=4-2 \epsilon$ to order $\epsilon^{0}$ and subsequently extended to all orders in $\epsilon$ in Ref. [7]. Two-loop corrections to this order were also obtained for massive quarks [8]. The three-loop form factors to order $\epsilon^{-1}$ (and $\epsilon^{0}$ for fermion loop contributions) were computed in $[3,9]$. One of the main motivations for obtaining analytical results for the form factors is the search for a deeper underlying structure of the coefficients, as proposed in Ref. [10] for planar box amplitudes.

In order to calculate the quark and gluon form factors at higher orders in perturbation theory, the amplitudes are reduced, by means of algebraic reduction procedures [12-15], to a small set of master integrals. At the three-loop level, the master integrals for massless form factors were identified in Ref. [16] and results for certain subsets are available in the literature [12,16-18]. Among the three-loop master integrals, the genuine three-loop vertex functions are the most challenging ones from a computational point of view. They correspond to two-particle cuts of the master integrals for massless four-loop off-shell propagator integrals [19], which have been used in the calculation of the scalar $R$-ratio [20]. The derivation of the three-loop vertex integrals is of comparable complexity to the four-loop propagator integrals.

Working in dimensional regularisation and expanding the master integrals in a Laurent series in $\epsilon$, the finite part of the three-loop form factors requires the extraction of all coefficients through transcendentality six, i.e. coefficients containing terms up to $\pi^{6}$ or $\zeta_{3}^{2}$. Note that the power of $\epsilon$ coming with coefficients of transcendentality six in the Laurent expansion is not always the same in the different master integrals: Transcendentality six can appear in the coefficients of the $\epsilon^{0}$-, $\epsilon$ - or $\epsilon^{2}$-terms in the Laurent series. If it appears in the $\epsilon^{k}$-term, this indicates that a prefactor $\sim 1 / \epsilon^{k}$ will come from the reduction to master integrals, such that an expansion up to transcendentality six of the master integrals will always be required.

Those genuine three-loop vertex functions which contain one-loop or two-loop propagator insertions were already given in Ref. [16]. The purpose of the present letter is to extend this calculation to all three-loop master integrals which have less than nine propagators. Each topology contains only one master integral, which is chosen to be the scalar integral, with no loop momenta in the numerator and with all propagators raised to unit power. It turns out that this subset of three-loop master integrals is sufficient in order to obtain the aforementioned fermion loop contributions within a Feynman diagrammatic approach [21]. At this point we would like to point out an error in Ref. [16], namely the basis of three-loop master integrals given there is too large, since certain two-particle cuts of four-loop propagator topologies [19] yield topologically identical three-loop vertex topologies. Consequently $A_{8,1}=A_{8,2} \equiv A_{8}$ and $A_{9,2}=A_{9,3}$. The corrected set of three-loop vertex integrals is given in Fig. 1 .

This letter is organised as follows. Computational methods to obtain analytical and nu- 
merical results of the three-loop vertex integrals with up to eight propagators are described in Section 2, and the analytical results for them are listed in Section 3. Section 4 contains our conclusions and an outlook.

\section{Master integrals: Classification and computational methods}

Vertex integrals with one off-shell and two on-shell legs and massless propagators depend only on one kinematic scale: the mass $q^{2}$ of the off-shell leg. The dependence on this scale is given by the mass dimension of the integral, such that the coefficients of the Laurent expansion in the dimensional regularisation parameter $\epsilon$ are real constants which are in general of increasing transcendentality in the Riemann $\zeta$-function, where the degree of transcendentality $(D T)$ is defined by

$$
\begin{aligned}
D T(r) & =0 \quad \text { for rational } r \\
D T\left(\pi^{k}\right) & =D T(\zeta(k))=k \\
D T(x \cdot y) & =D T(x)+D T(y) .
\end{aligned}
$$

At the three-loop level the quark form factor depends - like the process $e^{+} e^{-} \rightarrow 3$ jets at NNLO - on the following seven colour structures $[21,22]$

$$
N^{2}, \quad N^{0}, \quad 1 / N^{2}, \quad N_{F} \cdot N, \quad N_{F} / N, \quad N_{F}^{2}, \quad N_{F, \gamma},
$$

where the last colour factor stems from topologies in which the external gauge boson couples to a closed fermion loop. The three terms containing $N_{F}$ are referred to as fermionic corrections. They have been derived in Refs. [3,9] from the behaviour of the three-loop deep inelastic coefficient functions [11]. In the more conventional approach of computing multi-loop Feynman amplitudes the form factors are - after an algebraic reduction procedure [12-15] - expressed in terms of a small set of master integrals. It turns out [21] that the master integrals in Fig. 11 with at most eight propagators are sufficient in order to obtain the fermionic corrections to the form factor. The purpose of this letter is therefore to evaluate these master integrals. Those master integrals in Fig. 1 that contain single or multiple bubble insertions have already been computed in Ref. [16], the remaining ones with up to eight propagators - i.e. diagrams $A_{6,2}, A_{7,3}, A_{7,4}$, $A_{7,5}$, and $A_{8}$ - are subject of the present work. Working in dimensional regularisation with $D=4-2 \epsilon$, we give one of the diagrams $\left(A_{7,4}\right)$ in a closed form which is exact to all orders in $\epsilon$, containing $\Gamma$-functions and hypergeometric functions of unit argument. In all other cases we derive multiple - twofold to fourfold - Mellin-Barnes representations [23-26] from which the coefficients of the Laurent expansion in $\epsilon$ are obtained in an analytic form. As explained above, all coefficients through transcendentality six in the Riemann $\zeta$-function have to be included to obtain the finite part of the three-loop form factor.

For many practical applications, and to verify the analytical results, it is sufficient to know the numerical values of the coefficients in the Laurent expansion of the master integrals to some finite order. There are several techniques to obtain numerical values for the coefficients, one of them being the sector decomposition method, which is described in detail in Refs. [27, 28]. Using this technique, the Laurent expansions of all master integrals relevant to the three-loop 
form factors can be computed to, in principle, any desired order. In practice there are of course limitations, from CPU time for the numerical evaluation and from memory for the algebraic part of the sector decomposition procedure. The eight propagator graph $A_{8}$ is the most complex one from the sector decomposition point of view, not only due to the high number of propagators, but also because it exhibits spurious linear divergences at intermediate stages, which render the subtractions and thus the functions to be integrated more complicated. The computing time for $A_{8}$ up to order $\epsilon$ for a numerical precision of $0.1 \%$ is of the order of 4 hours on a $3.0 \mathrm{GHz} \mathrm{PC}$. For a precision of $1 \%$ the evaluation is more than 10 times faster.

Other methods of doing numerical cross checks, based on the numerical evaluation of MellinBarnes integrals, are provided by the packages in Refs. $[29,30]$, by means of which we were able to check most of the coefficients at the sub-permille level.

\section{Results}

In this section we list the results we obtained for the three-loop master integrals necessary for the fermionic corrections to the three-loop quark form factor, which are the diagrams $A_{6,2}, A_{7,3}$, $A_{7,4}, A_{7,5}$ and $A_{8}$ in Figure 1, All other diagrams with up to eight propagators possess so-called bubble insertions and have already been given in Ref. [16].

Diagram $A_{6,2}$

The first diagram to be considered is $A_{6,2}$. In Ref. [31] a representation of this diagram in terms of a one-dimensional integral over hypergeometric functions was given. Here we pursue a different strategy and derive a twofold Mellin-Barnes representation [24-26] from which the coefficients of the Laurent series expansion about $\epsilon=0$ can be computed. We start with

$$
A_{6,2}=\int \frac{d^{D} k}{(2 \pi)^{D}} \int \frac{d^{D} l}{(2 \pi)^{D}} \int \frac{d^{D} r}{(2 \pi)^{D}} \frac{1}{\left(k+p_{1}\right)^{2}\left(k+l-p_{2}\right)^{2} l^{2} r^{2}(r-k)^{2}(r-k-l)^{2}},
$$

and assume here and in the following that all propagators contain an infinitesimal $+i \eta$. We then derive the following expression that contains a triple integral over a Meijer-G function [32,33],

$$
\begin{aligned}
& A_{6,2}=-i S_{\Gamma}^{3}\left[-q^{2}-i \eta\right]^{-3 \epsilon} \frac{\Gamma^{3}(1-\epsilon) \Gamma(3 \epsilon)}{\Gamma(1-2 \epsilon) \Gamma(2-4 \epsilon)} \\
& \times \int_{0}^{1} d x d y d z x^{-\epsilon}(1-x)^{-3 \epsilon} y^{-\epsilon}(1-y)^{-3 \epsilon} z^{-2 \epsilon}(1-z)^{-2 \epsilon} \\
& \times G_{33}^{32}\left(x z+y(1-z) \mid \begin{array}{l}
\{-1+4 \epsilon,-1+4 \epsilon\},\{3 \epsilon\} \\
\{-1+3 \epsilon,-1+2 \epsilon, 0\},\{\}
\end{array}\right), \\
& \text { where } \quad q^{2}=\left(p_{1}+p_{2}\right)^{2} \quad \text { and } \quad S_{\Gamma}=\frac{1}{(4 \pi)^{D / 2} \Gamma(1-\epsilon)} \text {. }
\end{aligned}
$$

We now make use of the contour integral representation of the Meijer-G function [32,33], and subsequently decompose the argument by means of a second Mellin-Barnes representation. The 


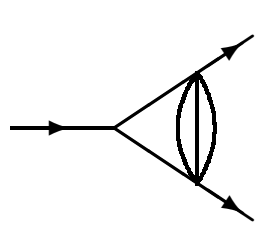

$A_{5,1}$

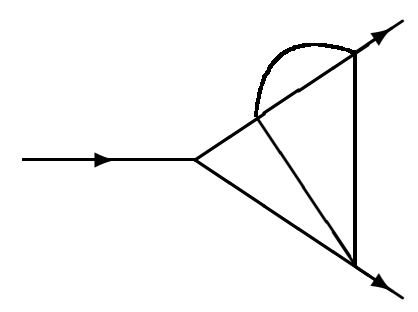

$A_{6,3}$

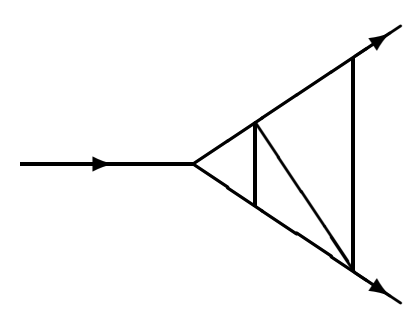

$A_{7,3}$

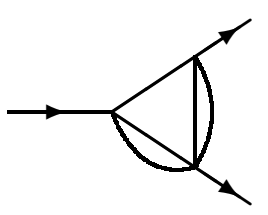

$A_{5,2}$

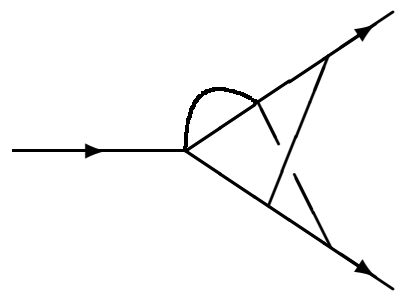

$A_{7,1}$

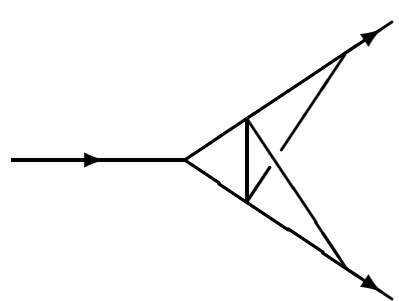

$A_{7,4}$

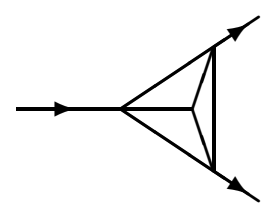

$A_{6,2}$

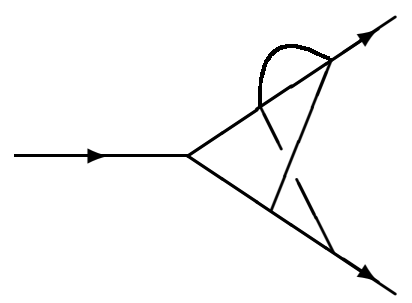

$A_{7,2}$

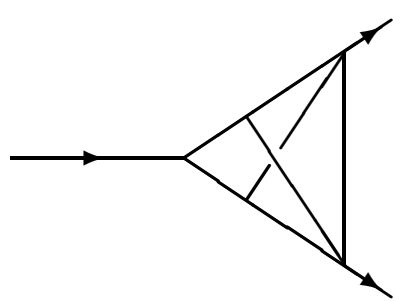

$A_{7,5}$

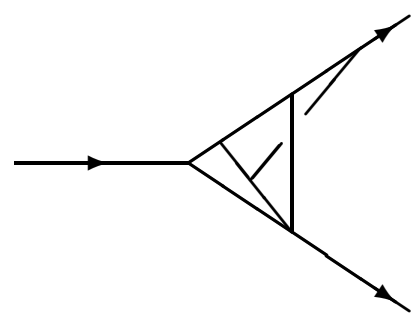

$A_{8}$

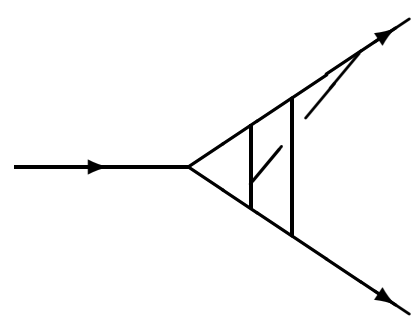

$A_{9,2}$

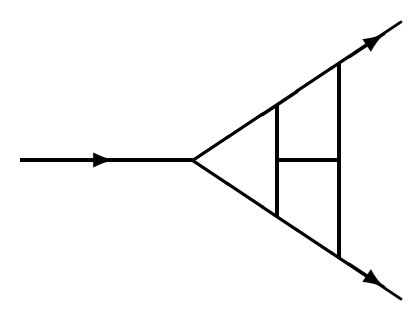

$A_{9,1}$

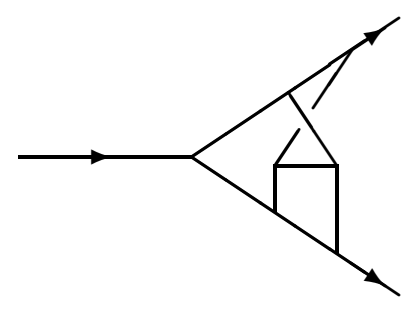

$A_{9,4}$

Figure 1: Three-loop master integrals with massless propagators. The incoming momentum is $q=p_{1}+p_{2}$. Outgoing lines are considered on-shell and massless, i.e. $p_{1}^{2}=p_{2}^{2}=0$. 
integrals over $x, y$, and $z$ can then be done in terms of $\Gamma$-functions. This leads to the following twofold Mellin-Barnes representation for $A_{6,2}$,

$$
\begin{aligned}
A_{6,2}= & -i S_{\Gamma}^{3}\left[-q^{2}-i \eta\right]^{-3 \epsilon} \frac{\Gamma^{3}(1-\epsilon) \Gamma(3 \epsilon) \Gamma^{2}(1-3 \epsilon)}{\Gamma(1-2 \epsilon) \Gamma(2-4 \epsilon)} \int_{c_{1}-i \infty}^{c_{1}+i \infty} \frac{d w_{1}}{2 \pi i} \int_{c_{2}-i \infty}^{c_{2}+i \infty} \frac{d w_{2}}{2 \pi i} \\
& \times \frac{\Gamma\left(-1+3 \epsilon-w_{1}\right) \Gamma\left(-1+2 \epsilon-w_{1}\right) \Gamma\left(2-4 \epsilon+w_{1}\right) \Gamma\left(-w_{2}\right) \Gamma\left(w_{2}-w_{1}\right)}{\Gamma\left(3 \epsilon-w_{1}\right) \Gamma\left(2-4 \epsilon+w_{2}\right) \Gamma\left(2-4 \epsilon+w_{1}-w_{2}\right)} \\
& \times \Gamma\left(1-\epsilon+w_{2}\right) \Gamma\left(1-\epsilon+w_{1}-w_{2}\right) \Gamma\left(1-2 \epsilon+w_{2}\right) \Gamma\left(1-2 \epsilon+w_{1}-w_{2}\right) .
\end{aligned}
$$

In the above equation (6) the contour integrals in the complex plane are along curves which separate left poles of $\Gamma$-functions from right ones, where "left poles" are poles stemming from a $\Gamma(\ldots+w)$ dependence, while "right poles" stem from a $\Gamma(\ldots-w)$ dependence [26]. The most convenient choice for these contours are straight lines parallel to the imaginary axis, i.e. the real parts along the curves are constant. According to Refs. [24,25], these real parts, together with the parameter $\epsilon$, must be chosen in such a way as to have positive arguments in all occurring $\Gamma$-functions in order to separate left and right poles in the desired way. One verifies easily that

$$
c_{1}=-\frac{6}{5}, \quad c_{2}=-\frac{1}{2}, \quad-\frac{1}{15}<\epsilon<\frac{3}{20}
$$

is an appropriate choice in Eq. (6) . From the fact that the origin lies within the allowed region for $\epsilon$, we conclude that the Mellin-Barnes integration does not produce any poles in $\epsilon$ in addition to the UV pole that is already present in the prefactor. Therefore the expansion in $\epsilon$ commutes with the contour integrations. Proceeding in this way, the Mellin-Barnes integrations can be done order by order in $\epsilon$. During this procedure, the contours can be closed at infinity to either side of the complex plane, and the corresponding residues are then summed with the appropriate global sign. To this end, the formulas in the Appendix of Ref. [26] prove extremely useful. The final result for $A_{6,2}$ is

$$
\begin{aligned}
A_{6,2}= & i S_{\Gamma}^{3}\left[-q^{2}-i \eta\right]^{-3 \epsilon} \\
\times & {\left[-\frac{2 \zeta_{3}}{\epsilon}-18 \zeta_{3}-\frac{7 \pi^{4}}{180}+\left(-122 \zeta_{3}-\frac{7 \pi^{4}}{20}+\frac{2 \pi^{2}}{3} \zeta_{3}-10 \zeta_{5}\right) \epsilon\right.} \\
& \left.+\left(-738 \zeta_{3}-\frac{427 \pi^{4}}{180}+6 \pi^{2} \zeta_{3}-90 \zeta_{5}+\frac{163 \pi^{6}}{7560}+76 \zeta_{3}^{2}\right) \epsilon^{2}+\mathcal{O}\left(\epsilon^{3}\right)\right] .
\end{aligned}
$$

In Ref. [31], two more orders of the $\epsilon$-expansion can be found.

Diagram $A_{7,3}$

We now turn our attention to the integral $A_{7,3}$. This integral will be represented, similarly to the integral $A_{6,2}$, as a multiple Mellin-Barnes integral:

$$
A_{7,3}=\int \frac{d^{D} k}{(2 \pi)^{D}} \int \frac{d^{D} l}{(2 \pi)^{D}} \int \frac{d^{D} r}{(2 \pi)^{D}} \frac{1}{k^{2}(k+q)^{2}\left(l-k-p_{2}\right)^{2}\left(l-p_{2}\right)^{2}(r+l)^{2} r^{2}\left(r-p_{1}\right)^{2}}
$$




$$
\begin{gathered}
=\quad i S_{\Gamma}^{3}\left[-q^{2}-i \eta\right]^{-1-3 \epsilon} \frac{\Gamma^{4}(1-\epsilon) \Gamma(-\epsilon)}{\Gamma(1-2 \epsilon) \Gamma(1-3 \epsilon)} \int_{c_{1}-i \infty}^{c_{1}+i \infty} \frac{d w_{1}}{2 \pi i} \int_{c_{2}-i \infty}^{c_{2}+i \infty} \frac{d w_{2}}{2 \pi i} \int_{c_{3}-i \infty}^{c_{3}+i \infty} \frac{d w_{3}}{2 \pi i} \frac{\Gamma\left(-w_{1}\right)}{\Gamma\left(1-w_{1}\right)} \\
\quad \times \frac{\Gamma\left(-3 \epsilon-w_{3}\right) \Gamma\left(1+2 \epsilon+w_{1}+w_{2}\right) \Gamma\left(1+w_{1}+w_{2}\right) \Gamma\left(-2 \epsilon-w_{2}\right) \Gamma\left(-\epsilon-w_{1}\right)}{\Gamma\left(1-3 \epsilon-w_{3}\right) \Gamma\left(2-2 \epsilon+w_{1}+w_{2}\right)} \\
\quad \times \Gamma\left(-w_{3}\right) \Gamma\left(\epsilon-w_{1}-w_{2}+w_{3}\right) \Gamma\left(1-\epsilon+w_{2}\right) \Gamma\left(1+w_{3}\right) \Gamma\left(-\epsilon+w_{1}-w_{3}\right) .
\end{gathered}
$$

The contour integrals are again along straight lines in the complex plane parallel to the imaginary axis, and as before we must choose the real parts of the integration variables such as to have positive arguments in all occurring $\Gamma$-functions. This is achieved by choosing

$$
c_{1}=-\frac{3}{20}, \quad c_{2}=-\frac{3}{5}, \quad c_{3}=-\frac{1}{2}, \quad-\frac{1}{8}<\epsilon<\frac{3}{20} .
$$

As it was the case for $A_{6,2}$, we have the origin within the allowed region for $\epsilon$ and therefore the Mellin-Barnes integration does not give rise to any additional poles in $\epsilon$, the only pole of the integral being the infrared pole that is already present in the prefactor in Eq. (9). We can thus again perform the contour integrations order by order in $\epsilon$. Since the leading coefficient turns out to have already transcendentality five, we only need to compute the first two terms in the expansion. They are given by

$$
A_{7,3}=i S_{\Gamma}^{3}\left[-q^{2}-i \eta\right]^{-1-3 \epsilon}\left[\left(-\frac{\pi^{2} \zeta_{3}}{6}-10 \zeta_{5}\right) \frac{1}{\epsilon}-\frac{119 \pi^{6}}{2160}-\frac{31}{2} \zeta_{3}^{2}+\mathcal{O}(\epsilon)\right] .
$$

During the evaluation of this integral we could not proceed until the end by merely applying Barnes Lemmata and corollaries thereof, but had to apply auxiliary integral representations of hypergeometric functions at intermediate steps. The benefit of this procedure is that it enables us to perform all Mellin-Barnes integrations, at the cost of introducing additional parameters over which we subsequently have to integrate. However, the integrations over these auxiliary parameters can be done in terms of logarithms and (harmonic) polylogarithms. Furthermore, we made extensive use of the package HPL $[34,35]$ and of an algorithm based on the nested sums approach $[36,37]$.

\section{Diagram $A_{7,4}$}

The next diagram we consider is $A_{7,4}$. At first glance it looks quite difficult since it lacks both a bubble insertion and a planar topology. However, it turns out to be simpler than the planar diagram $A_{7,3}$, and it can even be displayed in a closed form. The main reason for this is the fact that at the outer vertices of both outgoing lines only three lines meet, and hence the introduction of Feynman parameters allows for the combination of propagators that differ only by a light-like momentum. This property is absent in both $A_{6,2}$ and $A_{7,3}$, both of which did not reveal a closed form but only a multiple Mellin-Barnes representation. For $A_{7,4}$, we find

$$
A_{7,4}=\int \frac{d^{D} k}{(2 \pi)^{D}} \int \frac{d^{D} l}{(2 \pi)^{D}} \int \frac{d^{D} r}{(2 \pi)^{D}} \frac{1}{k^{2}(k-q)^{2}(r+l-k)^{2} l^{2}\left(l-p_{1}\right)^{2} r^{2}\left(r-p_{2}\right)^{2}}
$$




$$
\begin{aligned}
& =i S_{\Gamma}^{3}\left[-q^{2}-i \eta\right]^{-1-3 \epsilon} \cdot 2 \cdot \Gamma^{4}(1-\epsilon) \Gamma^{2}(-\epsilon) \\
& \times\left[\frac{\Gamma(1-\epsilon) \Gamma(3 \epsilon)}{(1-3 \epsilon)^{2} \Gamma(2-4 \epsilon)}{ }_{4} F_{3}(1,1-\epsilon, 1-3 \epsilon, 2-6 \epsilon ; 2-3 \epsilon, 2-3 \epsilon, 2-4 \epsilon ; 1)\right. \\
& -\frac{\Gamma(1-3 \epsilon) \Gamma(2-3 \epsilon) \Gamma(3 \epsilon) \Gamma(1+2 \epsilon)}{\Gamma(2-\epsilon) \Gamma(2-6 \epsilon)} \\
& \times{ }_{4} F_{3}(1,1,1+2 \epsilon, 2-3 \epsilon ; 2,2,2-\epsilon ; 1) \\
& +\frac{\Gamma^{2}(1-3 \epsilon) \Gamma(1+2 \epsilon) \Gamma(1+3 \epsilon)}{\Gamma(2-\epsilon) \Gamma(2-6 \epsilon)} \\
& \left.\times{ }_{4} F_{3}(1,1,1+2 \epsilon, 1+3 \epsilon ; 2,2,2-\epsilon ; 1)\right] \\
& =i S_{\Gamma}^{3}\left[-q^{2}-i \eta\right]^{-1-3 \epsilon} \\
& \times\left[\frac{6 \zeta_{3}}{\epsilon^{2}}+\left(\frac{11 \pi^{4}}{90}+36 \zeta_{3}\right) \frac{1}{\epsilon}+\left(\frac{11 \pi^{4}}{15}+216 \zeta_{3}-2 \pi^{2} \zeta_{3}+46 \zeta_{5}\right)\right. \\
& \left.+\left(\frac{22 \pi^{4}}{5}-\frac{19 \pi^{6}}{270}+1296 \zeta_{3}-12 \pi^{2} \zeta_{3}-282 \zeta_{3}^{2}+276 \zeta_{5}\right) \epsilon+\mathcal{O}\left(\epsilon^{2}\right)\right],
\end{aligned}
$$

where the expansion in $\epsilon$ was done by means of the Mathematica [38] package HypExp [31,39].

\section{Diagram $A_{7,5}$}

We now consider diagram $A_{7,5}$ for which we derive the following fourfold Mellin-Barnes representation.

$$
\begin{aligned}
A_{7,5}= & \int \frac{d^{D} k}{(2 \pi)^{D}} \int \frac{d^{D} l}{(2 \pi)^{D}} \int \frac{d^{D} r}{(2 \pi)^{D}} \frac{1}{k^{2}(k+q)^{2}(k+r)^{2}\left(l-p_{2}\right)^{2}(r-l)^{2} r^{2}\left(k+l+p_{1}\right)^{2}} \\
= & i S_{\Gamma}^{3}\left[-q^{2}-i \eta\right]^{-1-3 \epsilon} \frac{\Gamma^{3}(1-\epsilon)}{\Gamma(1-2 \epsilon) \Gamma(1-4 \epsilon)} \int_{c_{1}-i \infty}^{c_{1}+i \infty} \frac{d w_{1}}{2 \pi i} \int_{c_{2}-i \infty}^{c_{2}+i \infty} \frac{d w_{2}}{2 \pi i} \int_{c_{3}-i \infty}^{c_{3}+i \infty} \frac{d w_{3}}{2 \pi i} \int_{c_{4}-i \infty}^{c_{4}+i \infty} \frac{d w_{4}}{2 \pi i} \\
& \frac{\Gamma\left(w_{4}-w_{1}\right) \Gamma\left(1+w_{3}\right) \Gamma\left(-3 \epsilon-w_{3}\right) \Gamma\left(1-2 \epsilon+w_{1}+w_{2}-w_{4}\right) \Gamma\left(-w_{3}\right) \Gamma\left(-w_{4}\right)}{\Gamma\left(1-w_{1}+w_{3}+w_{4}\right)} \\
& \times \frac{\Gamma\left(1+3 \epsilon+w_{3}+w_{4}\right) \Gamma\left(1+\epsilon+w_{1}+w_{2}\right) \Gamma\left(1+w_{1}+w_{2}\right) \Gamma\left(-\epsilon-w_{1}\right) \Gamma\left(-\epsilon-w_{2}\right)}{\Gamma\left(2+\epsilon+w_{1}+w_{2}\right) \Gamma\left(2-2 \epsilon+w_{1}+w_{2}\right)} \\
& \times \frac{\Gamma\left(1-\epsilon+w_{2}\right) \Gamma\left(1+w_{3}\right) \Gamma\left(1-\epsilon+w_{1}\right) \Gamma\left(\epsilon-w_{1}-w_{2}+w_{3}+w_{4}\right) \Gamma\left(w_{4}-w_{2}\right)}{\Gamma\left(1-w_{2}+w_{3}+w_{4}\right)} .
\end{aligned}
$$


Like in the previous cases, the Mellin-Barnes integral does not generate poles in $\epsilon$, so we can therefore interchange the expansion in $\epsilon$ with the contour integrations. We choose

$$
c_{1}=-\frac{1}{5}, \quad c_{2}=-\frac{1}{4}, \quad c_{3}=-\frac{1}{7}, \quad c_{4}=-\frac{1}{11} .
$$

As before in the case of $A_{7,3}$ we can not proceed until the end by merely applying Barnes Lemmata and corollaries thereof, but again have to apply auxiliary integral and series representations at intermediate steps, this time even for a larger class of functions than before. Besides hypergeometric functions, these are mainly logarithms and (harmonic) polylogarithms as well as $\psi$-functions with

$$
\begin{aligned}
\psi^{(0)}(z) & =\frac{d}{d z} \ln [\Gamma(z)] \\
\psi^{(k)}(z) & =\frac{d}{d z} \psi^{(k-1)}(z) \text { for } \quad k=1,2, \ldots
\end{aligned}
$$

The sums and integrals over the auxiliary parameters are then performed by means of the same techniques as before. The final result for $A_{7,5}$ reads

$$
A_{7,5}=i S_{\Gamma}^{3}\left[-q^{2}-i \eta\right]^{-1-3 \epsilon}\left[2 \pi^{2} \zeta_{3}+10 \zeta_{5}+\left(12 \pi^{2} \zeta_{3}+60 \zeta_{5}+\frac{11 \pi^{6}}{162}+18 \zeta_{3}^{2}\right) \epsilon+\mathcal{O}\left(\epsilon^{2}\right)\right]
$$

\section{Diagram $A_{8}$}

The last diagram we consider is $A_{8}$ which can also be displayed as a fourfold Mellin-Barnes integral.

$$
\begin{aligned}
A_{8}= & \int \frac{d^{D} k}{(2 \pi)^{D}} \int \frac{d^{D} l}{(2 \pi)^{D}} \int \frac{d^{D} r}{(2 \pi)^{D}} \frac{1}{\left(k+p_{1}\right)^{2}(k+r)^{2}(k+r+q)^{2}(l-k)^{2}(l+r)^{2} l^{2} r^{2}\left(l+p_{1}\right)^{2}} \\
= & -i S_{\Gamma}^{3}\left[-q^{2}-i \eta\right]^{-2-3 \epsilon} \frac{\Gamma^{3}(1-\epsilon) \Gamma(-1-3 \epsilon)}{\Gamma(-2 \epsilon) \Gamma(-4 \epsilon)} \int_{c_{1}-i \infty}^{c_{1}+i \infty} \frac{d w_{1}}{2 \pi i} \int_{c_{2}-i \infty}^{c_{2}+i \infty} \frac{d w_{2}}{2 \pi i} \int_{c_{3}-i \infty}^{c_{3}+i \infty} \frac{d w_{3}}{2 \pi i} \int_{c_{4}-i \infty}^{c_{4}+i \infty} \frac{d w_{4}}{2 \pi i} \\
& \frac{\Gamma\left(1+w_{3}\right) \Gamma\left(1+w_{4}\right) \Gamma\left(w_{4}-w_{2}\right) \Gamma\left(w_{3}-w_{1}\right) \Gamma\left(-w_{4}\right) \Gamma\left(-w_{3}\right) \Gamma\left(2+w_{1}+w_{2}\right)}{\Gamma\left(2+w_{3}+w_{4}\right) \Gamma\left(1+w_{4}-w_{2}\right) \Gamma\left(1+w_{3}-w_{1}\right)} \\
& \times \frac{\Gamma\left(2+\epsilon+w_{1}+w_{2}\right) \Gamma\left(1-\epsilon+w_{1}\right) \Gamma\left(1-\epsilon+w_{2}\right) \Gamma\left(-1-\epsilon-w_{1}\right) \Gamma\left(-1-\epsilon-w_{2}\right)}{\Gamma\left(2-2 \epsilon+w_{1}+w_{2}\right) \Gamma\left(3+\epsilon+w_{1}+w_{2}\right)} \\
& \times \Gamma\left(2+3 \epsilon+w_{3}+w_{4}\right) \Gamma\left(1-2 \epsilon+w_{1}+w_{2}-w_{3}-w_{4}\right) \Gamma\left(\epsilon-w_{1}-w_{2}+w_{3}+w_{4}\right) .
\end{aligned}
$$


This time the Mellin-Barnes integral does indeed generate poles in $\epsilon$. We choose

$$
c_{1}=-\frac{7}{8}, \quad c_{2}=-\frac{19}{24}, \quad c_{3}=-\frac{13}{24}, \quad c_{4}=-\frac{25}{48}, \quad-\frac{5}{16}<\epsilon<-\frac{5}{24}
$$

in order to separate left poles of $\Gamma$-functions from right ones, and subsequently perform the analytic continuation to $\epsilon=0$ [29]. This generates four kernels, one four-dimensional one, two three-dimensional ones, and one two-dimensional one. We arrive at the final result

$$
\begin{gathered}
A_{8}=i S_{\Gamma}^{3}\left[-q^{2}-i \eta\right]^{-2-3 \epsilon}\left[\frac{8 \zeta_{3}}{3 \epsilon^{2}}+\left(\frac{5 \pi^{4}}{27}-8 \zeta_{3}\right) \frac{1}{\epsilon}+24 \zeta_{3}-\frac{5 \pi^{4}}{9}-\frac{52}{9} \pi^{2} \zeta_{3}+\frac{352}{3} \zeta_{5}\right. \\
\left.+\left(-72 \zeta_{3}+\frac{5 \pi^{4}}{3}+\frac{52}{3} \pi^{2} \zeta_{3}-352 \zeta_{5}+\frac{1709 \pi^{6}}{8505}-\frac{332}{3} \zeta_{3}^{2}\right) \epsilon+\mathcal{O}\left(\epsilon^{2}\right)\right] .
\end{gathered}
$$

Despite the fact that this integral has one more propagator compared to $A_{7,5}$ it was much simpler to evaluate than the former one, and we did not have to introduce any auxiliary parameters in integral or series representations. The reason is again that $A_{8}$ - contrary to $A_{7,5}$ - possesses an outgoing line with an outer vertex where only three lines meet.

\section{Conclusions and Outlook}

In this letter we have evaluated those master integrals for massless three-loop form factors which are necessary for the calculation of the fermionic corrections to the quark form factor. We obtained analytical results for all coefficients through transcendentality six in the Riemann $\zeta$-function, as required to obtain the finite part of the form factor at the three-loop level. For the integral $A_{7,4}$ we could obtain a representation which is valid to all orders in $\epsilon$, in terms of hypergeometric functions of unit argument. For the other integrals, we derived multiple Mellin-Barnes representations from which we extracted all necessary coefficients order by order in $\epsilon$.

The only missing pieces to complete the set of master integrals for massless three-loop form factors are therefore the three diagrams in Figure 1 which have nine propagators. It turns out that each of them can be expressed in terms of a sixfold Mellin-Barnes representation [40] which gives rise to $\mathcal{O}(100)$ single terms upon performing the analytical continuation to $\epsilon=0$ by means of the package MB [29]. Although the number of single integrals is quite large due to the extraction of high poles in $\epsilon$, and the evaluation is not completely automated at certain stages, the analytical results for these integrals are within reach [40].

\section{Acknowledgements}

We would like to thank Thomas Gehrmann and Beat Tödtli for communicating to us part of the structure of the three-loop form factor prior to publication. We thank Alejandro Daleo and David Kosower for useful discussions and for performing some cross checks. We would also like to thank Martin Beneke and Pietro Falgari for useful discussions. T.H. was supported by the Swiss National Science Foundation (SNF) and by Deutsche Forschungsgemeinschaft, 
SFB/TR 9 "Computergestützte Theoretische Teilchenphysik". The work of D.M. was supported by the Swiss National Science Foundation (SNF) under contract PBZH2-117028 and by the US Department of Energy under contract DE-AC02-76SF00515. The work of G.H. was supported by the UK Science and Technology Facilities Council. The authors would like to thank the Institute for Theoretical Physics at the University of Zürich, where most of this work was done.

\section{References}

[1] S. Catani, Phys. Lett. B 427 (1998) 161 hep-ph/9802439.

[2] G. Sterman and M.E. Tejeda-Yeomans, Phys. Lett. B 552 (2003) 48 hep-ph/0210130.

[3] S. Moch, J.A.M. Vermaseren and A. Vogt, JHEP 0508 (2005) 049 hep-ph/0507039.

[4] G. Kramer and B. Lampe, Z. Phys. C 34 (1987) 497; 42 (1989) 504(E);

T. Matsuura and W.L. van Neerven, Z. Phys. C 38 (1988) 623;

T. Matsuura, S.C. van der Maarck and W.L. van Neerven, Nucl. Phys. B 319 (1989) 570.

[5] R.V. Harlander, Phys. Lett. B 492 (2000) 74 hep-ph/0007289.

[6] V. Ravindran, J. Smith and W.L. van Neerven, Nucl. Phys. B 704 (2005) 332 hep-ph/0408315.

[7] T. Gehrmann, T. Huber and D. Maître, Phys. Lett. B 622 (2005) 295 hep-ph/0507061.

[8] W. Bernreuther et al., Nucl. Phys. B 706 (2005) 245 hep-ph/0406046; Nucl. Phys. B 712 (2005) 229 hep-ph/0412259]; Nucl. Phys. B 723 (2005) 91 hep-ph/0504190].

[9] S. Moch, J.A.M. Vermaseren and A. Vogt, Phys. Lett. B 625 (2005) 245 hep-ph/0508055.

[10] Z. Bern, L. J. Dixon and V. A. Smirnov, Phys. Rev. D 72 (2005) 085001 arXiv:hep-th/0505205.

[11] J.A.M. Vermaseren, A. Vogt and S. Moch, Nucl. Phys. B 724 (2005) 3 hep-ph/0504242.

[12] F.V. Tkachov, Phys. Lett. 100B (1981) 65;

K.G. Chetyrkin and F.V. Tkachov, Nucl. Phys. B192 (1981) 159.

[13] S. Laporta, Int. J. Mod. Phys. A 15 (2000) 5087 hep-ph/0102033.

[14] T. Gehrmann and E. Remiddi, Nucl. Phys. B 580 (2000) 485 hep-ph/9912329.

[15] C. Anastasiou and A. Lazopoulos, JHEP 0407 (2004) 046 hep-ph/0404258.

[16] T. Gehrmann, G. Heinrich, T. Huber and C. Studerus, Phys. Lett. B 640, 252 (2006) arXiv:hep-ph/0607185.

[17] S. Bekavac, hep-ph/0505174. 
[18] S.G. Gorishnii, S.A. Larin, L.R. Surguladze and F.V. Tkachov, Comput. Phys. Comm. 55 (1989) 381;

S.A. Larin, F.V. Tkachov and J.A.M. Vermaseren, NIKHEF-H-91-18.

[19] P.A. Baikov, Phys. Lett. B 634 (2006) 325 hep-ph/0507053.

[20] P.A. Baikov, K.G. Chetyrkin and J.H. Kühn, Phys. Rev. Lett. 96 (2006) 012003 hep-ph/0511063.

[21] T. Gehrmann and B. Tödtli, private communication

[22] A. Gehrmann-De Ridder, T. Gehrmann, E. W. N. Glover and G. Heinrich, JHEP 0711 (2007) 058. arXiv:0710.0346 [hep-ph]].

[23] V.A. Smirnov, Phys. Lett. B 460 (1999) 397 hep-ph/9905323.

[24] J.B. Tausk, Phys. Lett. B 469 (1999) 225 hep-ph/9909506.

[25] C. Anastasiou and A. Daleo, JHEP 0610 (2006) 031 arXiv:hep-ph/0511176.

[26] V. A. Smirnov, "Evaluating Feynman integrals", Springer Tracts Mod. Phys. 211 (2004) 1.

[27] T. Binoth and G. Heinrich, Nucl. Phys. B 585 (2000) 741 hep-ph/0004013.

[28] T. Binoth and G. Heinrich, Nucl. Phys. B 680 (2004) 375 hep-ph/0305234.

[29] M. Czakon, Comput. Phys. Commun. 175 (2006) 559 arXiv:hep-ph/0511200.

[30] J. Gluza, K. Kajda and T. Riemann, Comput. Phys. Commun. 177 (2007) 879 arXiv:0704.2423 [hep-ph]].

[31] T. Huber and D. Maitre, arXiv:0708.2443 [hep-ph].

[32] A. Erdélyi (ed.), Higher transcendental functions, Vol. 1, (McGraw-Hill, New York, 1953) .

[33] A. Erdélyi (ed.), Tables of Integral Transforms, Vol. 2, (McGraw-Hill, New York, 1954) .

[34] D. Maitre, Comput. Phys. Commun. 174 (2006) 222 arXiv:hep-ph/0507152.

[35] D. Maitre, arXiv:hep-ph/0703052.

[36] J. A. M. Vermaseren, Int. J. Mod. Phys. A 14 (1999) 2037 [arXiv:hep-ph/9806280].

[37] S. Moch, P. Uwer and S. Weinzierl, J. Math. Phys. 43 (2002) 3363 arXiv:hep-ph/0110083.

[38] MATHEMATICA 5.2, Copyright 2005 by Wolfram Research.

[39] T. Huber and D. Maître, Comput. Phys. Commun. 175 (2006) 122 hep-ph/0507094.

[40] T. Huber et. al., work in progress 